\title{
ADVOCACIA EM PROMOÇÃO DA SAÚDE: CONCEITOS, FUNDAMENTOS E ESTRATÉGIAS PARA A DEFESA DA EQUIDADE EM SAÚDE
}

\author{
Health promotion advocacy: concepts framework \\ and strategies to promote health equity
}

Ana Claudia Camargo Gonçalves Germani*

Fernando Aith**

\begin{abstract}
RESUMO
A advocacia em promoção da saúde representa um importante instrumento de participação social voltado à defesa do direito universal à saúde no Brasil. O objetivo do trabalho foi descrever os conceitos e fundamentos, bem como propor estratégias nessa área. A partir de revisão de literatura exploratória, desenvolveu-se um método de operacionalização das ações da advocacia em promoção da saúde. $\mathrm{O}$ argumento central, sintetizado em uma figura, propõe e descreve a pluralidade das estratégias para a concretização da advocacia em promoção da saúde. $O$ debate sobre os atores envolvidos procura apontar desdobramentos para as competências técnicas, necessárias sobretudo para profissionais. Acredita-se que o embasamento das práticas de advocacia em promoção da saúde possa fomentar o desenvolvimento/fortalecimento desse campo de atuação democrática, e dessa forma contribuir para o enfrentamento das iniquidades em saúde no Brasil.
\end{abstract}

Palavras-chave: Ação Intersetorial; Advocacia em Saúde; Competência Profissional; Promoção da Saúde.

(*) Doutora e mestre, Faculdade de Medicina, Universidade de São Paulo (FM/USP). Professora do Departamento de Medicina Preventiva, FM/USP. Médica. São Paulo/SP - Brasil. E-mail: accggermani@usp.br.

${ }^{\left({ }^{\star *}\right)}$ Doutor em Saúde Pública, Faculdade de Saúde Pública, Universidade de São Paulo (FSP/ USP); Mestre em Direito, Faculdade de Direito, Universidade de São Paulo (FD/USP). Professor do Departamento de Medicina Preventiva, FM/USP. Advogado. São Paulo/SP - Brasil. E-mail: fernando.aith@usp.br.

Texto recebido em: 18/07/2012. Revisado em: 09/10/2012. Nova revisão em: 29/10/2012. Aprovado em: 30/10/2012. 


\begin{abstract}
Advocacy in health promotion is an important instrument of social participation aimed to defend the universal right to health in Brazil. The objective is to describe the concepts and fundamentals and propose strategies in this area. From a literature review (domestic and foreign) exploratory, developed a method of advocacy actions operational in health promotion, in order to assist the practice in this field. The central argument, summarized in a Figure, proposes and describes the variety of strategies for the implementation of advocacy in health promotion. The debate about the actors involved tries to point out consequences for the technical skills needed especially for professionals. It is believed that the foundation of the practice of advocacy in health promotion could stimulate the development / strengthening of democratic playing field, and thus contribute to tackling the health inequalities in Brazil.
\end{abstract}

Keywords: Health Advocacy; Health Promotion; Intersectorial Action; Professional Competence.

\title{
Introdução
}

A advocacia em promoção da saúde é um tema ainda pouco explorado tanto no campo do direito quanto nos campos da saúde pública e da medicina. O empenho na discussão de conceitos e fundamentos teóricos sobre o tema possibilita a ampliação e a renovação das estratégias e práticas necessárias para a solução de questões complexas e coletivas que envolvem a saúde. ${ }^{(1)}$

Os conceitos, fundamentos e estratégias aqui apresentados evidenciam a estreita relação entre as dimensões técnica, ética e política das ações em defesa do direito à saúde, em especial no que se refere à promoção da saúde. Nesse sentido, o presente artigo busca discutir de forma aprofundada o tema da advocacia em promoção da saúde, tendo como objetivo principal ampliar as capacidades de defesa da equidade em saúde em nossa sociedade.

\section{Conceitos básicos para a advocacia em promoção da saúde}

A advocacia em promoção da saúde representa hoje, nas sociedades democráticas, um exercício de cidadania essencial para o desenvolvimento social da nação, tendo como objetivos a equidade em saúde e a garantia do bem-estar físico, mental e social das pessoas. Trata-se de uma atividade que democratiza e difunde conhecimentos e evidências científicas sobre as melhores formas de

1 AYRES, J.R.C.M. Norma e formação: horizontes filosóficos para as práticas de avaliação no contexto da promoção da saúde. Ciência \& Saúde Coletiva, Rio de Janeiro, v. 9, n. 3, p. 583-592, 2004. 
se promover a saúde em uma determinada sociedade, em conformidade com o conceito de saúde vigente nesta mesma sociedade.

A promoção da saúde ocupa um papel cada vez mais relevante no campo da saúde pública, razão pela qual vem ganhando espaço no direito sanitário brasileiro à medida que motiva a produção legislativa em saúde e determina uma parte significativa das políticas públicas de saúde desenvolvidas pelo Estado. No campo legislativo, a promoção da saúde consta como um dever específico do Estado no Art. 196 da Constituição Federal, que dispõe expressamente que a saúde é direito de todos e dever do Estado, devendo ser garantida mediante políticas econômicas e sociais que visem ao acesso universal e igualitário às ações e serviços de promoção da saúde.(2)

A advocacia em promoção da saúde representa, nesse contexto, um importante instrumento de participação social voltado à defesa do direito universal à saúde no Brasil. Para compreender as estratégias e objetivos da advocacia em promoção da saúde em uma sociedade democrática, convém, preliminarmente, tecer algumas reflexões introdutórias sobre os principais conceitos e contextos que cercam o tema.

\section{Saúde - direito de todos e dever do Estado: conceitos de saúde}

A saúde é uma experiência universal, familiar a todos, mas um olhar criterioso revelará que o conceito de saúde é complexo, podendo ser visto a partir de perspectivas diversas.

Uma fotografia simplificada da extensa literatura sobre o assunto permite identificar duas vertentes principais. A primeira é chamada (bio)médica e inclui teorias que focam o corpo e visualizam, grosso modo, a saúde e a doença como opostos entre si. Já a segunda perspectiva parte da premissa do processo dinâmico entre situações de saúde e doença e é socialmente orientada, ou seja, valoriza a determinação social do processo saúde-doença-cuidado.

O conceito de saúde mais difundido hoje é o proposto pela Organização Mundial da Saúde (OMS) no preâmbulo de sua Constituição, de 1946: "saúde é o estado de completo bem-estar físico, mental e social, e não somente a ausência de doenças ou enfermidades". (3) A amplitude de tal conceito, inicialmente percebida como fator positivo, atualmente esbarra em críticas por possibilitar interpretações tidas como idealistas ou sem aplicação prática.

\footnotetext{
${ }^{2}$ Art. 196. BRASIL. Constituição da República Federativa do Brasil de 1988. Disponível em: <http:// www.planalto.gov.br/ccivil_03/Constituicao/ConstituicaoCompilado.htm>. Acesso em: 29 mar. 2013.

${ }^{3}$ ORGANIZAÇÃO MUNDIAL DA SAÚDE. Constituição de 1946. Disponível em: <http://apps.who.int/gb/

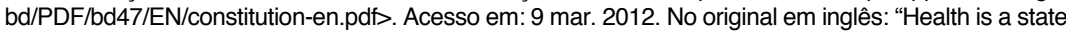
of complete physical, mental and social well-being and not merely the absence of disease or infirmity".
} 
Entre as reformulações propostas, a Carta de Ottawa ${ }^{(4)}$ coloca que a "saúde deve ser vista como um recurso para a vida cotidiana, e não como objetivo de viver. Nesse sentido, a saúde é um conceito positivo, que enfatiza recursos sociais e pessoais, bem como capacidades físicas". Nessa ótica, o conceito de saúde e a sua proteção como direito dependem, em sociedades democráticas, da necessária participação ativa de todos os sujeitos envolvidos no processo saúde-doença-cuidado, incluindo usuários do sistema de saúde, profissionais da saúde, gestores e representantes de setores como educação, segurança, meio ambiente, direito e muitos outros. ${ }^{(5)}$

Embora o conceito de saúde mostre-se polissêmico e derive necessariamente do contexto social onde é discutido, o reconhecimento da saúde como direito possibilitou grandes avanços no sentido da efetiva promoção da saúde no mundo.

No âmbito internacional, a Constituição da OMS de 1946 marcou o reconhecimento da saúde como direito humano universal, fundamental para a dignidade do ser humano, e traçou as linhas gerais para a sua proteção. Vinte anos depois, o Pacto Internacional dos Direitos Econômicos, Sociais e Culturais de 1966 consolidou a ideia da saúde como um direito fundamental a ser protegido pelos Estados, definindo algumas ações fundamentais que devem ser adotadas pelos Estados para a proteção do direito humano fundamental à saúde. ${ }^{(6)}$ Desde então a Organização das Nações Unidas (ONU), diretamente ou por meio da OMS, vem organizando uma série de encontros internacionais voltados ao aperfeiçoamento do sistema internacional de proteção do direito à saúde, tais como as Conferências Internacionais de Promoção da Saúde, ou ainda a recente Declaração do Rio de 2011.

No Brasil, o reconhecimento formal da saúde como direito deu-se somente com a promulgação da Constituição de 1988, que expressamente reconhece a saúde como direito social (Art. $6^{\circ}$, caput), direito de todos e dever do Estado (Art. 196), a ser garantido mediante políticas sociais e econômicas que visem ao acesso universal e igualitário às ações e serviços para sua promoção, proteção e recuperação.

O reconhecimento expresso da saúde como direito veio acompanhado, portanto, da compreensão de que a sua garantia somente será possível se houver ações

${ }^{4}$ CARTA DE OTTAWA. Documento resultante da Primeira Conferência Internacional Sobre Promoção da Saúde de novembro de 1986. Disponível em: <http://www.opas.org.br/promocao/ uploadArq/Ottawa.pdf>. Acesso em: 12 de mar. 2012.

5 JOHANSSON, H.; WEINEHALL, L.; EMMELIN, M. "It depends on what you mean": a qualitative study of Swedish health professionals' view on health and health promotion. BMC Health Services Research, v. 9, p. 191, 2009.

${ }^{6}$ ORGANIZAÇÃO DAS NAÇÕES UNIDAS. Pacto Internacional dos Direitos Econômicos, Sociais e Culturais. Disponível em: <http://www2.mre.gov.br/dai/m_591_1992.htm>. Acesso em: 12 mar. 2012. 
e serviços voltados à promoção da saúde. Nesse sentido é que foi aprovada a Política Nacional de Promoção da Saúde (PNPS), por meio da Portaria $n^{\circ} 687$ MS/GM. ${ }^{(7)}$

O direito à saúde representa um importante fator de promoção da saúde, à medida que estimula o aperfeiçoamento permanente do direito sanitário para que este seja capaz de estabelecer obrigações e fixar diretrizes de atuação dos cidadãos e do Estado para a promoção e equidade em saúde.

A partir do reconhecimento da saúde como direito de todos e, principalmente, como dever do Estado, faz-se necessário que a sociedade, por meio de seus cidadãos e grupos organizados, atue em defesa do direito à saúde, cobrando do Estado as suas obrigações constitucionais. Como visto, o reconhecimento da saúde como um direito implica o dever estatal de garantir o acesso universal e igualitário às ações e serviços de promoção de saúde. Esse cenário torna fundamental o desenvolvimento de ferramentas de defesa desse aspecto específico de garantia do direito à saúde, defesa essa que deve ser realizada, nas sociedades democráticas, por meio da advocacia em promoção da saúde.

\section{Promoção da saúde: campos de ação}

A promoção da saúde pode ser definida como o "processo de capacitação das pessoas e comunidades para modificarem os determinantes da saúde em benefício da própria qualidade de vida". ${ }^{(8)}$ Ou ainda, conforme consta da Carta de Ottawa:

Promoção da saúde é o nome dado ao processo de capacitação da comunidade para atuar na melhoria de sua qualidade de vida e saúde, incluindo uma maior participação no controle deste processo. Para atingir um Estado de completo bem-estar físico, mental e social os indivíduos e grupos devem saber identificar aspirações, satisfazer necessidades e modificar favoravelmente o meio ambiente... Assim, a promoção da saúde não é responsabilidade exclusiva do setor saúde, e vai além de um estilo de vida saudável, na direção de um bem-estar global..(9)

O mesmo documento aponta três estratégias básicas da promoção da saúde: a capacitação, a mediação e a advocacia. Tendo em vista que a promoção da saúde envolve capacitação, uma das principais estratégias encontradas para promover a saúde é a da educação em saúde. Esta pode ser entendida como

\footnotetext{
${ }^{7}$ BRASIL. MINISTÉRIO DA SAÚDE. Política Nacional de Promoção da Saúde. Disponível em: <http:// bvsms.saude.gov.br/bvs/publicacoes/politica_nacional_promocao_saude_3ed.pdf $>$. Acesso em: 13 jul.2012.

${ }^{8}$ CANDEIAS, N.M.F. Conceitos de educação e de promoção em saúde: mudanças individuais e mudanças organizacionais. Revista de Saúde Pública, São Paulo, v. 31, n. 2, p. 209-13, abr. 1997.

${ }^{9}$ CARTA DE OTTAWA. Documento resultante da Primeira Conferência Internacional Sobre Promoção da Saúde de novembro de 1986, cit.
} 
"quaisquer combinações de experiências de aprendizagem delineadas com vistas a facilitar ações voluntárias conducentes à saúde". ${ }^{(10)}$ Assim, a educação em saúde é um dos caminhos para a promoção da saúde. Vale destacar que alguns autores usam os termos promoção da saúde e educação em saúde como se fossem intercambiáveis, enquanto outros enfatizam as diferenças de abordagem, princípios e efetividade de cada um.

No entanto, como bem percebido na Carta de Ottawa, a promoção da saúde abrange várias estratégias possíveis, que vão além do setor saúde e, naturalmente, vão além da educação em saúde. Nesse sentido, a mediação de esforços interssetoriais faz-se primordial e, ao mesmo tempo, permite considerar como promoção da saúde quaisquer atividades do Estado e da sociedade que facilitem o cuidado com a saúde, por meio de ações sociais ou políticas públicas que promovam o acesso universal e igualitário à água potável, ao saneamento básico, a alimentos saudáveis, a produtos de higiene, dentre outros.

Nesse sentido, a advocacia em saúde emerge como um instrumento fundamental para a promoção da saúde em Estados democráticos, à medida que possibilita para o indivíduo e para os diversos grupos sociais que compõem a sociedade o debate livre, amplo, aberto e plural sobre as diferentes formas de se promover a saúde, debate este que tem como pano de fundo, como visto, a reflexão sobre o próprio conceito de saúde que deve vigorar em uma sociedade.

O texto, produzido no Canadá e reforçado nas demais conferências, apresenta cinco campos de prática da promoção da saúde, que por sua expressiva importância foram resumidos a seguir: 1) elaboração de políticas públicas saudáveis, que apontem para a equidade em saúde; 2) criação de ambientes favoráveis, como forma de fortalecer a abordagem socioecológica da saúde; 3) reforço da ação comunitária, isto é, a participação efetiva e concreta da comunidade na eleição de prioridades, na tomada de decisões e na elaboração e desenvolvimento de estratégias para alcançar melhor nível de saúde; 4) reorganização dos serviços de saúde (com mudanças na educação e formação profissional); e 5) desenvolvimento de habilidades pessoais para manutenção da autonomia nas diferentes etapas da vida e no enfrentamento das enfermidades. ${ }^{(11)}$

\section{Advocacia em promoção da saúde: conceito e abrangência}

A partir do reconhecimento da saúde como direito surge uma correlação estreita entre a promoção da saúde e o direito, em especial o direito sanitário. E é justamente

\footnotetext{
${ }^{10}$ CANDEIAS, N.M.F. op.cit.

${ }^{11}$ BRASIL. MINISTÉRIO DA SAÚDE. Secretaria de Políticas de Saúde. Projeto Promoção da Saúde. As Cartas da Promoção da Saúde. Ministério da Saúde, Secretaria de Políticas de Saúde, Projeto Promoção da Saúde - Brasília: Ministério da Saúde, 2002. Disponível em: <http://bvsms.saude.gov. br/bvs/publicacoes/cartas_promocao.pdf>. Acesso em: 13 jul.2012.
} 
da integração do direito sanitário com a promoção da saúde que podemos extrair o conceito e a abrangência da advocacia em promoção da saúde.

Sob a ótica jurídica, a advocacia em saúde refere-se ao processo de reivindicação de direitos até então não respeitados, por indivíduos ou grupos, visando influir na definição ou na implementação de uma política pública mediante estratégias legais e éticas. ${ }^{(12)}$ A advocacia em saúde não se limita, portanto, a ações judiciais e demandas encaminhadas ao Poder Judiciário. A atividade de advocacia em saúde abrange um conjunto diversificado de ações voltadas à defesa do direito à saúde e representa uma atividade complexa, desenvolvida por um indivíduo ou por um grupo de pessoas, que podem envolver os demais poderes (Legislativo e Executivo).

A advocacia em saúde contribui diretamente, em diferentes momentos e circunstâncias, para a vivência democrática e construção de um diálogo e de um consenso no espaço público. Trata-se, nos dizeres de Zioni, "de um conjunto de procedimentos que permitem a divulgação de informações e uma mobilização, ou envolvimento, de diferentes grupos sociais". (13) Nesse sentido, a prática da advocacia em saúde garante a divulgação e o conhecimento de diferentes concepções de saúde, que podem e devem coexistir em sociedades plurais e democráticas.

A Constituição de 1988 abriu espaço para a ampliação da advocacia em saúde no Brasil, conforme bem apontado por Torronteguy e Raupp:

Reivindicar um direito, ou seja, advogar, é uma atividade que pode acontecer em quatro situações distintas: em um primeiro plano, a advocacia é a reivindicação pelo reconhecimento de determinado direito, vale dizer, pela sua positivação no ordenamento jurídico. Em um segundo nível, quando o direito já houver sido reconhecido, a advocacia pode direcionar-se à criação de garantias para o direito reconhecido. Em terceiro lugar, por vezes, existem tanto o direito como as suas garantias, mas falta-lhes, por alguma razão, efetividade. Enfim, é possível ainda que a dificuldade política esteja na necessidade de derrogação ou alteração de uma lei vigente. ${ }^{(14)}$

Conceito bastante semelhante acerca da advocacia em saúde aparece em documentos norteadores da promoção da saúde, cartas de conferências internacionais, glossário de promoção da saúde da OMS e na própria PNPS, e é sintetizado por Canel e Castro.

\footnotetext{
${ }^{12}$ DALLARI, S.G.; BARBER-MADDEN, R.; TORRES-FERNANDES, M.C.; SHUQUAIR, N.S.M.S.A.Q.; WATANABE, H.A. Advocacia em saúde no Brasil contemporâneo. Revista de Saúde Pública, São Paulo. v. 30, n. 6, p. 592-601, abr. 1996.

${ }^{13}$ ZIONI, F. A. Advocacia em Saúde e sua Contribuição à Democracia. Revista de Direito Sanitário, São Paulo, v. 9, n. 1, p. 70-73, mar./jun. 2008.

${ }_{14}$ TORRONTEGUY, M.A.A.; RAUPP, L. Obstáculos Políticos à Advocacia Sanitária no Brasil: A pesquisa com Células-Tronco Embrionárias. Revista de Direito Sanitário, São Paulo. v. 9, n. 1, p. 86-106, mar./jun. 2008.
} 
A advocacia em saúde é constituída por ações de indivíduos ou de grupos organizados - os chamados atores sociais - que procuram influir sobre autoridades e sobre particulares, para reivindicar direitos na área da saúde, principalmente em benefício da parcela da população menos favorecida. ${ }^{(15)}$

Dados os conceitos expostos, podemos definir a advocacia em promoção da saúde como o conjunto coordenado e articulado de ações de cidadãos e/ou grupos sociais, voltado a influir sobre as autoridades estatais e sobre a sociedade em geral para promoção do bem-estar físico, mental e social dos indivíduos e da comunidade, para a garantia da equidade em saúde.

Percebe-se, pelo conceito exposto, que a advocacia em promoção da saúde envolve um conjunto de atores, saberes e fazeres que devem ser articulados na sociedade democrática para a finalidade específica de promoção da saúde, englobando, assim, elaboração e implementação de políticas públicas, ações de educação em saúde que apoiem modos de vida, desenvolvimento de ambiente saudável (domiciliar, profissional, comunitário), oferta de serviços com foco para a saúde, dentre outros temas interssetoriais fundamentais para a proteção do direito à saúde.

Um importante ponto que deve ser analisado para a compreensão da abrangência da advocacia em promoção da saúde refere-se à identificação dos atores principais desse tipo de atividade no ambiente social. Dallari et al. ${ }^{(16)}$ e Canel e Castro ${ }^{(17)}$ apontam que, no contexto da advocacia em saúde, o advogado inclui os bacharéis em direito, os profissionais de saúde e, sobretudo, todo cidadão-cidadã que deseje reivindicar seu direito à saúde. É oportuno destacar que a advocacia em saúde entendida aqui como prática lato sensu pode e deve contar com a advocacia judiciária tradicional (stricto sensu) como uma importante estratégia de ação e também como fonte de retroalimentação contínua.

Já em 1994, Feuerwerker ${ }^{(18)}$ reflete sobre o papel das equipes de saúde na advocacia em saúde quando descreve a experiência de mobilização contra falta de água e melhores condições de saneamento iniciada pelos profissionais de uma unidade básica de saúde. Andrade et al. ${ }^{(19)}$ identificam na literatura da América Latina e Caribe publicações que reforçam o exercício de advocacia em saúde, com foco no bem-estar da criança, na atuação de profissionais de saúde.

${ }^{15}$ CANEL, R.C.; CASTRO, C.G.J. A Advocacia em saúde como uma estratégia para a promoção da Saúde. Revista de Direito Sanitário, São Paulo, v. 9, n.1, p. 74-85, mar./jun. 2008.

${ }^{16}$ DALLARI, S.G.; BARBER-MADDEN, R.; TORRES-FERNANDES, M.C.; SHUQUAIR, N.S.M.S.A.Q.; WATANABE, H.A. op.cit.

${ }^{17}$ CANEL, R.C.; CASTRO, C.G.J. op.cit.

${ }^{18}$ FEUERWERKER, L.C.M. O papel das equipes de saúde na advocacia em saúde. Saúde em Debate, Rio de Janeiro, v. 45, p. 47-25, 1994.

${ }^{19}$ ANDRADE, R.D.; MELLO, D.F.; SILVA, M.A.I.; VENTURA, C.A.A. Advocacia em saúde na atenção à criança: revisão de literatura. Revista Brasileira de Enfermagem, Brasília, v. 64, n. 4, p. 738-44, 2011. 
Pode-se dizer que, atualmente, a força de trabalho potencial em promoção da saúde é composta por uma ampla gama de profissionais com formações diversas, ultrapassando as 14 profissões da saúde regulamentadas pela $R e-$ solução $n^{\circ} 287^{(20)}$ do Conselho Nacional de Saúde e as inúmeras ocupações definidas pelo Código Brasileiro de Ocupações, que em 1998 somavam mais de 40 categorias. ${ }^{(21)}$ Importante apontar que poucos países normatizam/regulamentam a promoção da saúde como uma profissão, com exercício e condições de trabalho definidos.

Embora a promoção da saúde esteja inserida no contexto das diferentes profissões, a formação em promoção da saúde ao longo dos cursos de graduação ainda é incipiente. No Brasil, crescem as oportunidades de formação complementar em promoção da saúde, na forma de cursos breves, congressos, simpósios até atividades estendidas por um ano ou mais (mestrados e doutorados).

De toda sorte, Carlisle(22) coloca que o conceito em si de advocacia pode ser desnecessariamente intimidador, mas defende que se trata de formar cidadãos e, na lógica do presente artigo, formar profissionais, incluindo os relacionados à área da saúde, para que sejam agentes de mudança, para que nutram o desejo de "fazer a diferença".

Tal ponto é reforçado por Galer-Unti et al. ${ }^{(23)}$ e Shilton ${ }^{(24)}$ quando destacam que, apesar de os documentos norteadores da promoção da saúde reconhecerem a advocacia em saúde como prioridade, o assunto ainda é pouco desenvolvido no campo das práticas. Caira et al. ${ }^{(25)}$ destacam que os próprios "promotores de saúde" também reconhecem a importância da advocacia em promoção da saúde; contudo, não a exercem. Algumas justificativas descritas na literatura são: a falta de tempo, outras prioridades, frustração com o processo, escassez de recursos e dúvidas sobre o impacto de ações individuais ${ }^{(26)}$.

${ }^{20}$ CONSELHO NACIONAL DE SAÚDE. Resolução $n^{\circ} 287$, de 8 de outubro de 1998. "Relaciona 14 (quatorze) categorias profissionais de saúde de nível superior para fins de atuação no CNS: assistentes sociais, biólogos, biomédicos, profissionais de educação física, enfermeiros, farmacêuticos, fisioterapeutas; fonoaudiólogos, médicos, médicos veterinários, nutricionistas, odontólogos, psicólogos e terapeutas ocupacionais". Disponível em: <http://conselho.saude.gov.br/ resolucoes/reso_98.htm>. Acesso em: 29 mar. 2013.

${ }^{21}$ GIRARDI, S. N.; FERNANDES JUNIOR, H.; CARVALHO, C. L. A regulamentação das profissões de saúde no Brasil. Espaço para Saúde, Londrina, v. 2, n. 1. Disponível em: <http://www.ccs.uel.br/ espaçoparasaude/v2n1/RPSB.htm>. Acesso em: 13 jul. 2012.

${ }^{22}$ CARLISLE, S. Health promotion, advocacy and health inequalities: a conceptual framework. Health Promotion International, Oxford, v. 15, n. 4, p. 369-376, 2000.

${ }^{23}$ GALER-UNTI, R.A.; TAPPE, M.K.; LACHENMAYR, S. Advocacy 101: Getting Started in Health Education Advocacy. Health Promotion Practice, Thousand Oaks, v. 5, p. 280-.288, 2004.

${ }^{24}$ SHILTON T. Advocacy for physical activity-from evidence to influence. Global Health Promotion, v. 13, n. 2, p. 118-126, 2006.

${ }^{25}$ CAIRA, N.M.; LACHENMAYR, S.; SHEINFELD, J.; GOODHART, F.W.; CANCIALOSI, L.; LEWIS, C. The Health Educator's Role in Advocacy and Policy: Principles, Processes, Programs, and Partnerships. Health Promotion Practice, Thousand Oaks, v. 4, p. 303-313, 2003.

${ }^{26}$ GALER-UNTI, R.A.; TAPPE, M.K.; LACHENMAYR, S. op. cit. 
Como resposta e com o título provocativo "da evidência para a influência", Shilton ${ }^{(27)}$ coloca o desafio de traduzir a dimensão científica das evidências para as práticas dos profissionais, da comunidade e, sobretudo, dos tomadores de decisão no contexto político. Galer-Unti et al., ${ }^{(28)}$ por sua vez, propõem a "fluência" dos promotores da saúde na linguagem da advocacia em saúde.

De forma complementar à proposição anterior, Caira et al. ${ }^{(29)}$ apostam que espaços e processos de formação e discussão sobre os princípios da advocacia em promoção da saúde sejam decisivos para tal "fluência" dos profissionais envolvidos. Ainda na arena internacional, defende-se que a advocacia em promoção da saúde é uma competência requerida globalmente tanto dos cidadãos quanto dos profissionais de saúde, apesar das diferenças culturais, sociais, econômicas e políticas.

A partir das convergências descritas, fica claro que as contribuições específicas do setor saúde (focado na promoção da saúde) e do direito apresentam fronteiras sobrepostas e permitem a flexibilização dos papéis profissionais. Nesse sentido, o reconhecimento da interdependência de saberes valoriza o potencial interprofissional e intersetorial das práticas, ou seja, as ações de advocacia em promoção da saúde ganham força com o contato entre as diferentes categorias profissionais, sobretudo quando integrados.

Em contrapartida, é possível antever certas dificuldades para tal integração. Casos de lobby e a própria judicialização da saúde (sobretudo da assistência médico-farmacêutica) mostram que, muitas vezes, existem interesses conflitantes entre a promoção da saúde, a advocacia em saúde ${ }^{(30)} \mathrm{e}$ as decisões judiciais. ${ }^{(31)} \mathrm{A}$ concentração de ações individuais envolvendo tratamentos e/ou medicamentos, ou ainda a reinvindicação de acesso às tecnologias não incorporadas pelo Sistema Único de Saúde (SUS), conflitam por vezes, com as necessidades (individuais e coletivas) relacionadas à promoção da saúde e a prevenção de doenças.

Importante pontuar, mesmo que de forma breve, que tal panorama dos interesses comerciais e econômicos influencia (e também é influenciado) a mídia. A "era" das Tecnologias de Informação e Comunicação (TICs), marcada sobretudo pela internet, transformou as formas de disseminação de informações especialmente relacionadas à saúde. A funcionalidade crescente, a facilidade de uso, a

\footnotetext{
${ }^{27}$ SHILTON, T. op.cit.

${ }^{28}$ Id. Ibid.

${ }^{29}$ CAIRA, N.M.; LACHENMAYR, S.; SHEINFELD, J.; GOODHART, F.W.; CANCIALOSI, L.; LEWIS, C. op.cit.

${ }^{30}$ CHIEFI, A.L.; BARATA, R.B. Judicialização da política pública de assistência farmacêutica e equidade. Cadernos de Saúde Pública, Rio de Janeiro, v. 25, n. 8, p. 1839-1849, jun. 2009.

${ }^{31}$ MACHADO, F.R.S. Contribuições ao debate da judicialização da saúde no Brasil. Revista de Direito Sanitário, São Paulo, v. 9, n. 2, p. 73-91, jul./out. 2008.
} 
diversidade de informações estimulam o uso da rede $w w w$ por profissionais e leigos. ${ }^{(32)}$ Estudo australiano recente mostra que 551 (69\%) das pessoas entrevistadas por telefone havia tido, visto, lido ou ouvido alguma(s) informação(ões) sobre saúde na mídia nos últimos 12 meses. ${ }^{(33)}$

Como contraponto, os pesquisadores do referido estudo questionam a qualidade, a precisão e a neutralidade da abordagem midiática, bem como a necessidade de validar as informações obtidas com fontes seguras, científicas. Em suas conclusões, colocam que a proliferação de oportunidades de se informar torna ainda maior o desafio de assegurar pessoas bem informadas.

Claro está que o cenário atual é multifacetado, e no presente artigo adotou-se como norte o aprimoramento da prática da advocacia em promoção da saúde, nas atividades de cidadania e profissionais, baseada em evidências científicas voltadas a ações eficazes e efetivas. Nesse sentido, apresentam-se a seguir, de forma objetiva, as diferentes estratégias possíveis de serem adotadas para a promoção da saúde no âmbito de um Estado Democrático de Direito.

Para tanto, o método de investigação utilizado foi a revisão narrativa, de natureza qualitativa. Nesse sentido, procurou-se reunir o conhecimento atual (nacional e estrangeiro) sobre as estratégias de advocacia em promoção da saúde. ${ }^{\left({ }^{(4)}\right.}$ A busca de referências foi realizada entre agosto de 2011 e setembro de 2012, na base de dado SciELO, utilizando os descritores: "advocacia em saúde" e "promoção da saúde", e na base PubMed, usando a palavra "advocacy" e o termo "MeSH (Medical Subject Headings) health promotion". Foram analisados textos em português e inglês. Foram incluídos artigos relevantes, independente de sua data de publicação.

\section{Estratégias de advocacia em promoção da saúde}

A prática da advocacia em promoção da saúde exige o conhecimento das diferentes estratégias possíveis de serem adotadas em um Estado Democrático de Direito. Cada estratégia pode ser entendida como uma arte: a arte de aplicar com efetividade os recursos de que se dispõe, ou de explorar as condições favoráveis de que porventura se desfrute, visando ao alcance de determinados objetivos.

Com base no atual modelo democrático de organização do Estado e da sociedade, podemos identificar algumas das principais estratégias atualmente existentes para o advogado em promoção da saúde defender a equidade em saúde e a promoção do bem-estar físico, mental e social dos indivíduos.

32 SOARES, M.C. Internet e saúde: possibilidades e limitações. Textos de la CiberSociedad, 4. Temática Variada. 2004. Disponível em: <http://www.cibersociedad.net/textos/articulo.php?art=51>. Acesso em: 15 out. 2012.

${ }^{33}$ HOGUE, M-C.B.; DORAN, E.; HENRY, D.A. A prompt to the web: the media and health information seeking behaviour. PLoS ONE, v. 7, n. 4, p. e34314, 2012.

${ }^{34}$ ROTHER, E.T. Revisão sistemática x revisão narrativa. Acta Paul Enferm., São Paulo, v. 20, n. 2, p. v-vi, 2007. 
A Figura 1, adaptada de modelo proposto por Carlisle, ${ }^{(35)}$ integra as diferentes estratégias e ao mesmo tempo explicita a operacionalização do conceito da advocacia em promoção da saúde.

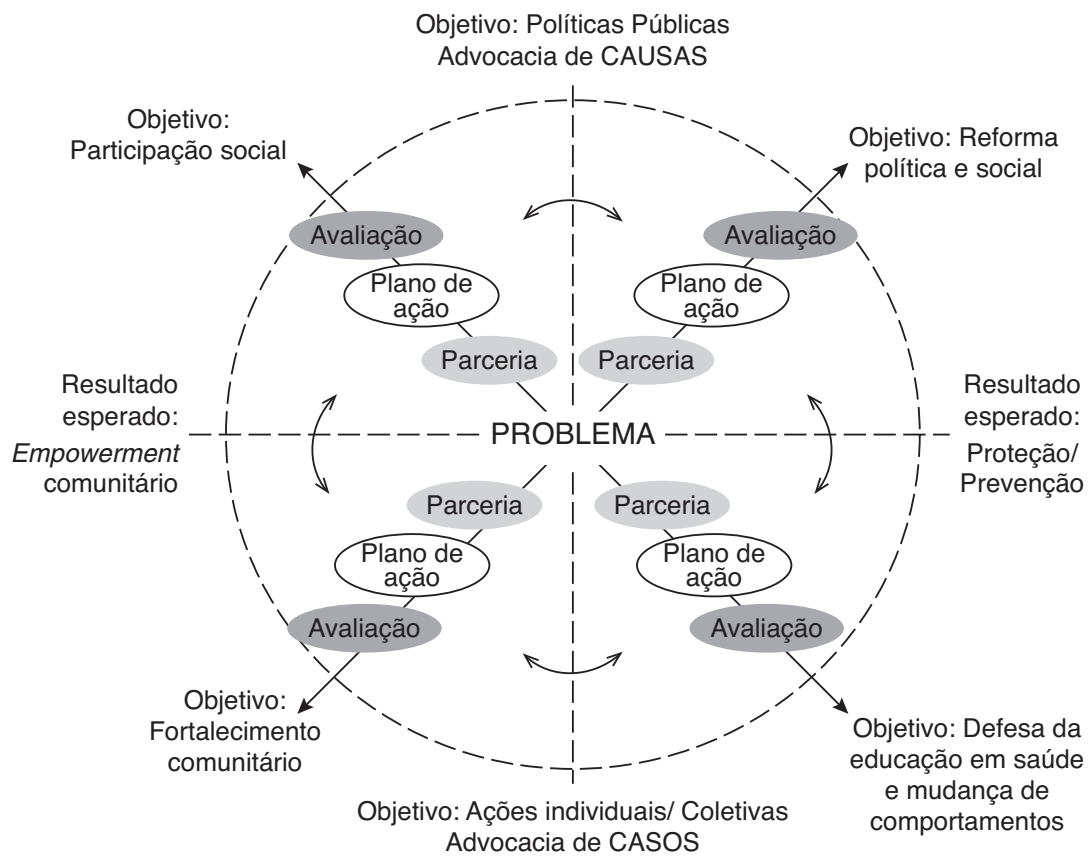

Figura 1. Estratégias de advocacia em promoção da saúde

A partir da identificação do problema a ser enfrentado, que situamos no centro da Figura 1, duas coordenadas são destacadas: o objetivo que se pretende com a ação de advocacia em promoção da saúde a ser realizado e os resultados concretos esperados.

Com relação aos objetivos, eles podem ter seu foco na "causa", entendida como as condições normativas ou de políticas públicas que possuem relação direta e influenciam o problema identificado; ou nos "casos", entendidos como aspectos individuais e coletivos mais específicos do problema, que podem ser enfrentados com ações pontuais a serem adotadas pelo indivíduo e/ou pelas coletividades (famílias, empresas ou grupos).

\footnotetext{
${ }^{35}$ CARLISLE, S. op.cit.
}

R. Dir. sanit., São Paulo, v. 14, n. 1, p. 34-59, mar./jun. 2013 
No que se refere aos resultados concretos esperados, estes podem ser o "empowerment" social, aqui entendido como "um processo e um resultado de ações que afetam a distribuição de poder no âmbito das esferas pessoais, intersubjetivas e políticas"; (36) ou a proteção e prevenção, entendida como uma ação específica de saúde voltada à redução do risco de doenças e outros agravos de saúde (individual e coletiva).

Importante notar que a complexidade dos problemas relacionados à promoção da saúde fatalmente exigirá a formulação de estratégias de advocacia em saúde diversificadas, capazes de abordar o problema em todas as suas dimensões. Nesse sentido, os objetivos e os resultados esperados não devem ser entendidos como polarizados ou mutuamente excludentes. O percurso tracejado na Figura 1 marca que os eixos em si apenas sustentam um continuum de possibilidades para o desenvolvimento de práticas da advocacia em promoção da saúde.

\section{Caracterização do problema e coleta de informações}

Inicialmente, é necessário caracterizar o problema a ser enfrentado (ponto central do modelo), tendo como ponto de partida a busca da equidade em saúde, um dos aspectos essenciais da promoção da saúde pontuados na PNPS. Esta política ainda destaca em suas diretrizes o estímulo a ações intersetoriais, o fortalecimento da participação social e o esforço para mudanças organizacionais.

A PNPS priorizou como ações específicas para o biênio 2006-2007:(37) alimentação saudável, prática corporal/atividade física, prevenção e controle do tabagismo, redução da morbimortalidade em decorrência do uso abusivo de álcool e outras drogas, redução da morbimortalidade por acidentes de trânsito, prevenção da violência e estímulo à cultura de paz, promoção do desenvolvimento sustentável.

A política fixada no âmbito nacional sugere ainda que as intervenções de promoção da saúde tenham como objetivo os problemas e as necessidades de saúde e seus determinantes, definidos pela Comissão Nacional sobre os Determinantes Sociais da Saúde (CNDSS) como "os fatores sociais, econômicos, culturais, étnicos/raciais, psicológicos e comportamentais que influenciam a ocorrência de problemas de saúde e seus fatores de risco na população."(38) Em outras palavras, a identificação de problemas relacionados à promoção da saúde exige o pensar sobre a "causa das causas".

\footnotetext{
${ }^{36}$ CARVALHO, S.R. Os múltiplos sentidos da categoria "empowerment" no projeto de promoção à saúde. Cadernos de Saúde Pública, Rio de Janeiro, v. 20, n. 4, p. 1088-1095, jul./ago. 2004.

${ }^{37}$ Trata-se da recomendação ainda vigente.

38 Ainda há um debate sobre a melhor terminologia - determinantes de saúde, determinantes sociais de saúde ou determinantes de iniquidade em saúde - de toda forma, adotamos o termo determinantes sociais compreendendo sua amplitude e profundidade.
} 
Nesse sentido, a análise do problema de interesse deve ser direcionada pelo acrônimo Pest ${ }^{(39)}$ (Político, Econômico, Social e Tecnológico) ou SLPET (sendo a letra $L$ relacionada a aspectos legais). Em outras palavras, cabe detalhar qual o contexto político relacionado, quais as possíveis variáveis econômicas, sociais e tecnológicas, uma vez que as informações consistentes sobre o problema são suporte para a tomada de decisões. (Não obstante, cabe a ressalva de que a pesquisa aqui defendida tem um porte diferente quando comparada à pesquisa científica em seu modelo tradicional.)

Assim, a advocacia em promoção da saúde exige a conciliação/integração de conhecimentos técnicos sobre o processo saúde-doença-cuidado com conhecimentos políticos e jurídicos associados ao tema, tais como as políticas públicas em andamento, a legislação incidente sobre o tema, as discussões que estão sendo travadas no Congresso Nacional ou nos parlamentos dos entes federativos, bem como o entendimento do Poder Judiciário sobre o tema.

O Anexo 1 apresenta alguns endereços eletrônicos que permitem o acesso à legislação nacional, estadual e municipal - vigente e em discussão (consulta pública) - considerados como instrumental básico para a advocacia em saúde como um todo. São elencados também exemplos de materiais usados (nacionais e estrangeiros) para a formação em advocacia em promoção da saúde.

O reconhecimento do problema a ser enfrentado e da estrutura jurídica a ele relacionada permite identificar o direito (assegurado ou não por lei) que está sendo violado e as eventuais omissões estatais para a garantia desse direito. Feito isso, torna-se possível traçar o objetivo da ação de advocacia em promoção da saúde, a partir de duas questões fundamentais: o que deve ser feito para controlar/eliminar o problema? Quais os resultados que se pode esperar com a ação de advocacia em saúde específica a ser desenvolvida?

\section{Definição dos objetivos da advocacia em promoção da saúde}

Em geral, os objetivos apontam para a(s) solução(ões) possível(is) aos problemas caracterizados. Sendo a promoção da saúde um tema relacionado com a saúde pública, é muito comum que dentre os objetivos da advocacia em promoção da saúde figurem a criação, a alteração ou o fim de uma política pública governamental específica. Também podem figurar como objetivos o estabelecimento, por meio de leis (Poder Legislativo) e regulamentos (Poder Executivo), de diretrizes e procedimentos a serem considerados pelo indivíduo, família, empresas e Estado para fins de promoção da saúde e redução das iniquidades. Descreve-se, então, como tais objetivos circundam os polos da Figura 1.

\footnotetext{
${ }^{39}$ Public Health Alliance for the Island of Ireland (PHAII). Public Health Advocacy Toolki, Belfast, 2007. 68p. Disponível em: <http://www.phaii.org/>. Acesso em: 11 jan. 2012.
} 
Dallari et al. ${ }^{(40)}$ mencionam que o objetivo da advocacia em saúde pode recair sobre a reivindicação de direitos de um indivíduo ou um grupo de pessoas, como por exemplo nos casos de advocacia de interesses coletivos ou difusos, ou podem estar ligados ao cumprimento das normas existentes; ou, ainda, podem estar ligados à necessidade de alteração de uma lei ou de cumprimento de uma lei já vigente, isto é, a advocacia legislativa.

$\mathrm{Na}$ área específica da advocacia em promoção da saúde, partindo-se, por exemplo, do acidente de trabalho com material cortante entre catadores de material reciclável (problema), o objetivo da ação pode transitar entre o direito de um único (ou de um grupo) catador relacionado ao uso de luvas específicas [Equipamento de Proteção Individual (EPI)] - foco nos casos - ou a proposição de normatização específica sobre proteção individual para essa categoria profissional - foco nas causas.

Carlisle(41) coloca que a advocacia para a educação em saúde e mudança de comportamentos de indivíduos e grupos pode ser uma forma de redução das iniquidades (na realidade brasileira, podemos mencionar como exemplo o direito a medicações para tratamento de dependência química ao tabaco). Outra possibilidade, também no tocante aos casos, é a defesa de direitos de coletivos cerceados por limitações, como cidadãos com agravos mentais ou outras incapacidades. Já as ações voltadas para as causas reforçam a necessidade de reformas políticas. Tendo em vista a diversidade de proposições, as ações podem versar tanto sobre a regulação de produtos prejudiciais à saúde (como tabaco, álcool e drogas ilícitas) como também abordar o enfrentamento de determinantes sociais (políticas para redução de desemprego, estímulo à distribuição de renda). De toda forma, esse nível (quadrante) enfatiza o caráter intersetorial da promoção da saúde.

Cabe destacar que, em um Estado democrático, um dos principais enfoques da reforma política deve ser o aprimoramento dos mecanismos de participação efetiva da população na definição de normas jurídicas e de políticas públicas. Tendo em vista o movimento proposto na Figura 1, vale, nesse momento, detalhar objetivos da advocacia em promoção da saúde voltados para o "empowerment" comunitário. O entendimento de tal categoria como um contínuo, isto é, com um componente individual que interage com coletivos e por sua vez influencia e é influenciado pelo contexto macro, permite pensar ações que incluem desde o fortalecimento de grupos até o exercício substantivo da participação social. ${ }^{(42)}$

\footnotetext{
${ }^{40}$ DALLARI, S.G.; BARBER-MADDEN, R.; TORRES-FERNANDES, M.C.; SHUQUAIR, N.S.M.S.A.Q.; WATANABE, H.A. op.cit.

${ }^{41}$ CARLISLE, S. op.cit.

${ }^{42}$ CARVALHO, S.R. op. cit.
} 


\section{Articulação de apoios e parcerias para a advocacia em promoção da saúde}

Caracterizado o problema e compreendida qual a solução possível ou, mais provavelmente, quais caminhos apoiam o enfrentamento do problema, é preciso pensar (e agir) na criação de uma rede de "stakeholders", conceito amplamente usado no contexto empresarial, aqui traduzido e expandido para a ideia de pessoas chave.

Em síntese, sugere-se que seja feita uma lista de pessoas e organizações/ instituições que são influenciadas pelo tema e/ou tenham influência e interesse. Importante o cuidado em detectar pessoas com interesse, ou seja, que atribuem importância ao tema e pessoas influentes, pessoas com poder de ação, reconhecidas como "autoridade", considerando potenciais aliados e também possíveis adversários. Convém lembrar que, além das pessoas diretamente interessadas e influentes, é importante mobilizar a opinião pública e, em especial, formadores de opinião que ainda não se posicionaram sobre o assunto. ${ }^{(43)}$

Cabe lembrar que no Brasil duas portarias ${ }^{(44),(45)}$ oficializam um Comitê Gestor da PNPS (CGPNPS), que tem como atribuições consolidar a PNPS e sua agenda nacional, articular e integrar as ações de promoção da saúde no contexto do Pacto pela Saúde, incentivar a inclusão do tema nos planos municipais e estaduais, além de coordenar e monitorar a implementação da PNPS.

O Comitê conta com representantes (e suplentes) de todas as secretarias do Ministério da Saúde, suas fundações, agências, Conselho Nacional de Secretários Municipais de Saúde (Conasems) e Conselho Nacional de Secretários Estaduais de Saúde (Conass). Com efeito, esse grupo figura como um interlocutor oficial em relação à PNPS que merece atenção para ações da advocacia em promoção da saúde. Uma característica desse Comitê que chama a atenção é a falta de representação da sociedade (organizações não governamentais, academia, conselhos profissionais etc.), demonstrando o distanciamento ainda existente entre o Estado e a sociedade no que se refere à promoção da saúde no país.

A identificação das pessoas chave pede ainda o resgate da discussão sobre a intersetorialidade. A efetividade de ações de advocacia em promoção da saúde demanda alianças colaborativas entre setores, instituições e atores de diferentes segmentos. $O$ mapeamento das pessoas chave deve ser seguido, então, por parcerias que demonstrem as convergências de esforços e a sinergia

\footnotetext{
${ }^{43}$ ASSOCIAÇÃO PARANAENSE DA PARADA DA DIVERSIDADE - APPAD. Guia de advocacy e prevenção em HIVIAIDS: gays e outros homens que fazem sexo com homens. Curitiba. 2009. 101p. Disponível em: <http://www.abglt.org.br/docs/interagir_guia.pdf>. Acesso em 12 mar. 2012.

${ }^{44}$ BRASIL. Ministério da Saúde. Portaria n 1.409, de 13 de junho de 2007. "Institui o Comitê Gestor da Política Nacional de Promoção da Saúde". Disponível em: < http://portal.saude.gov.br/portal/ arquivos/pdf/portaria_1409_comite_gestor.pdf>. Acesso em: 29 mar. 2013.

${ }^{45}$ BRASIL. Ministério da Saúde. Portaria $n^{\circ} 68$, de 24 de junho de 2008. Disponível em: <http://bvsms. saude.gov.br/bvs/saudelegis/svs/2008/prt0068_24_06_2008.html>. Acesso em: 29 mar. 2013.
} 
de olhares, recursos e expertises, em diferentes níveis. Como resume Carlisle, a advocacia em promoção da saúde é uma atividade multinível, multissetorial e multidisciplinar. ${ }^{(46)}$

\section{Planejamento e execução da advocacia em promoção da saúde}

Passa-se, então, à fase de planejamento de estratégias de advocacia em promoção da saúde. Publicações nacionais de organizações não governamentais ${ }^{(47),(48)}$ reforçam que após a pesquisa e o estabelecimento de parcerias é preciso definir qual a mensagem a ser divulgada. Tendo em vista o que se deseja defender (objetivo transformado em mensagem), pode-se pensar em como fazê-lo. Para tanto, Shilton et al. delimitam cinco núcleos: mobilização dos profissionais, das organizações e/ou instituições/serviços, da comunidade, da mídia e dos tomadores de decisão/políticos. ${ }^{(49)}$

Como dito, a promoção da saúde é um campo aberto a práticas interprofissionais; contudo, a "força de trabalho" envolvida em tais ações muitas vezes não reconhece a sua potência. Por exemplo, as práticas de atividade física envolvem diferentes profissionais em diferentes setores (saúde, educação, esportes e recreação), sendo, muitas vezes, necessários esforços para engajar tal grupo e mobilizá-lo de maneira que sua voz seja ampliada e demande publicamente maior atenção ao incremento do tempo de atividade física, para seguir no exemplo. Portanto, um primeiro plano de trabalho pode concentrar-se na mobilização dos profissionais diretamente envolvidos com o problema.

Outro foco é a advocacia em promoção da saúde praticada para a reorientação de organizações, isto é, o uso de conhecimentos e técnicas que estimulem e mobilizem mudanças internas, estruturais, de processos e de prioridades das próprias organizações envolvidas. Mais uma vez usando como modelo a atividade física e reforçando a intersetorialidade: o setor de esportes poderia sugerir em suas políticas e diretrizes a participação de atletas em eventos de caminhada e recreação; a área de transportes, por sua vez, priorizaria os transportes coletivos, as vias de caminhada e as ciclovias. Já o setor de planejamento daria prioridade a projetos acessíveis, que estimulassem escolhas fisicamente ativas.

Há ainda ações de advocacia em promoção da saúde com foco na participação da comunidade, de maneira esclarecida, consciente, voluntária e organizada. É

\footnotetext{
${ }^{46}$ CARLISLE, S. op.cit.

${ }^{47}$ ASSOCIAÇÃO PARANAENSE DA PARADA DA DIVERSIDADE - APPAD. Guia de advocacy e prevenção em HIVIAIDS: gays e outros homens que fazem sexo com homens, cit.

${ }^{48}$ COALITION QUÉBÉCOI POUR LE CONTRÔLE DU TABAC. Fazendo pesquisa para advocacy: a arte de converter o impossível no inevitável - e com baixo custo. 2006. 44p. Disponível em: <http:// actbr.org.br/uploads/conteudo/49_860_TABA.pdf>. Acesso em: 12 mar. 2012.

${ }^{49}$ SHILTON, T. op.cit.
} 
essencial assegurar que as pessoas saibam como defender os recursos necessários para a execução da ação de promoção da saúde desejada.

Além das matrizes de mobilização profissional e comunitária, defende-se a advocacia em promoção da saúde envolvendo a mídia (impressa, televisiva, sonora, eletrônica).(50) Uma vez consensuada e definida qual a mensagem a ser defendida - respeitando a acurácia científica e o propósito que se deseja alcançar -, é necessário selecionar e utilizar os métodos de comunicação apropriados para o grupo-alvo específico; deve-se ainda ponderar sobre o melhor momento (quando divulgar).

Por fim, outro cenário considerado central é a esfera política. Como sinalizado na declaração da 5ª Conferência Internacional de Promoção da Saúde sediada no México ${ }^{(51)}$, a promoção da saúde deve ser "prioridade fundamental das políticas e programas locais, regionais, nacionais e internacionais." Para tanto, Shilton et al. ${ }^{(52)}$ e Galer-Unti et al. ${ }^{(53)}$ reconhecem a importância e possibilidade de influência sobre políticos e governantes, seja no processo eleitoral (campanhas ou voto), seja na tomada de decisões (petições públicas).

\section{Avaliação de práticas de advocacia em promoção da saúde}

Finalmente, uma importante estratégia de advocacia em promoção da saúde refere-se à avaliação das diferentes práticas de advocacia em promoção da saúde realizadas em uma determinada sociedade, para se identificarem seus principais acertos, erros e resultados e, com base nisso, aperfeiçoar as próprias estratégias de advocacia existentes.

Como exemplo, as ações de advocacia em promoção da saúde relacionadas à prevenção do HIV/AIDS no Brasil são constantemente mencionadas como exitosas, à medida que de fato orientaram a sociedade brasileira e o Estado no sentido da redução dos riscos individuais e coletivos de infecção, transmissão e desenvolvimento da doença. A advocacia em saúde realizada por organizações não governamentais que representam doentes e familiares, por profissionais de saúde, por acadêmicos, dentre outros atores relevantes, certamente foi o fio condutor para o desenvolvimento da Política Nacional de HIV/AIDS e para a incorporação, nessa política, da promoção da saúde como estratégia fundamental.

A implementação e o cumprimento das medidas preconizadas pela Convenção-Quadro para o Controle do Tabaco (primeiro tratado internacional de

\footnotetext{
50 GALER-UNTI, R.A. Advocacy 2.0: advocating in the digital age. Health Promotion Practice, Thousand Oaks, v. 11, n. 6, p. 784-787, 2010.

${ }^{51}$ BRASIL. MINISTÉRIO DA SAÚDE. Secretaria de Políticas de Saúde. Projeto Promoção da Saúde. As Cartas da Promoção da Saúde, cit.

${ }^{52}$ SHILTON, T. op.cit.

${ }^{53}$ GALER-UNTI, R.A.; TAPPE, M.K.; LACHENMAYR, S. op.cit.
} 
saúde pública da história da OMS) ${ }^{(54)}$ também serve como modelo de práticas de sucesso no Brasil. O país, além de coordenar o processo de elaboração durante os anos de 1999 e 2003, teve sua adesão formalmente ratificada pelo Senado Federal em 27 de outubro de 2005. Desde então, a Política Nacional de Controle do Tabaco ${ }^{(55)}$ avança em ações de promoção da saúde, prevenção de doenças relacionadas e tratamento da dependência química (e suas consequências), sendo apoiada de forma importante pela sociedade civil.

Outras atividades de advocacia em promoção da saúde podem ser mapeadas no Brasil e no mundo, mas ainda há uma carência de trabalhos científicos e estudos que compilem e avaliem essas ações, no sentido de verificar quais os principais elementos que fazem com que uma atividade específica de advocacia em promoção da saúde resulte na adoção, pela sociedade e pelo Estado, de práticas eficientes no campo da promoção da saúde.

Embora a avaliação da eficácia das diferentes estratégias de advocacia em saúde ainda seja um tema pouco explorado, vale lembrar, como exemplo de um esforço nesse sentido, o trabalho de Rodney et al., no qual os autores propuseram três tipos de abordagens para avaliar ações de advocacia em saúde envolvendo trabalhadores engajados com comunidades indígenas nos Estados Unidos. Os autores elaboraram questionários para avaliar: a) o treinamento dos trabalhadores, b) a percepção da comunidade (na figura de gerentes dos serviços) e c) perspectiva dos clientes. ${ }^{(56)}$ Iniciativas nesse sentido são bastante relevantes para que as ações de defesa da saúde em sociedades democráticas sejam cada vez mais eficazes e abrangentes.

Autores estadunidenses preconizam que os trabalhos de avaliação da advocacia em promoção da saúde adotem uma postura de aprendizado contínuo, isto é, um processo de reconhecimento das estratégias que vêm funcionando e daquelas que não estão, de forma que sejam realizados os ajustes necessários. Enfatizam que a avaliação não deve ser uma atribuição de determinado setor, mas sim uma contribuição de todos os envolvidos no planejamento da ação de advocacia. Outros direcionamentos apresentados seguem no sentido de aferir ou registrar: a) o grau de organização da comunidade envolvida, b) a presença ou emergência de movimentos sociais (ou de classe) e c) a presença de lideranças locais. ${ }^{(57)}$

\footnotetext{
${ }^{54}$ WORLD HEALTH ORGANIZATION (WHO). An International Framework Convention for Tobacco Control. Fact Sheet $n^{\circ}$ 160. Disponível em: <http://www.who.int/inf-fs/en/fact160.html>. Tradução disponível em: <http://actbr.org.br/pdfs/CQCT.pdf>. Acesso em: 13 jul. 2012.

${ }^{55}$ BRASIL. Instituto Nacional do Câncer INCA. Observatório da política nacional de controle do tabaco. Disponível em: <http://www2.inca.gov.br/wps/wcm/connect/observatorio_controle_tabaco/ site/status_politica/a_politica_nacional>. Acesso em: 12 mar. 2012.

${ }^{56}$ RODNEY, M.; CLASEN, C.; GOLDMAN, G.; MARKERT, R.; DEANE, D. Three evaluation methods of a community health advocate program. Journal of Community Health, v. 23, n. 5, Oct. 1998.

${ }^{57}$ DEVLIN-FOLTZ, FAGEN, M.C.; REED, E.; MEDINA, R.; NEIGER, B.L. Advocacy Evaluation: challenges and emerging trends. Health Promotion Practice, Thousand Oaks, v. 13, n. 5, p. 581-586, 2012.
} 


\section{Desenvolvimento e organização de domínios e competências profissionais no campo da advocacia em promoção da saúde}

Diante do exposto e como nos mostra a Figura 1, na advocacia em promoção da saúde não existe um único jeito ou a maneira certa de atuar. $O$ enfrentamento das iniquidades em saúde pede a composição de um contínuo de ações, que considerem e articulem os binômios sujeito/coletivo, público/privado, Estado/ sociedade, clínica/política e setor sanitário/outros setores. ${ }^{(58)}$ Tal pluralidade das práticas só é possível graças à diversidade de profissionais envolvidos, influenciados por seus contextos e por suas formações.

Nesse aspecto, Carlisle coloca que o papel do profissional envolvido com a advocacia em promoção da saúde no eixo da proteção/prevenção é marcado pela representação, ou seja, espera-se a atuação direta de "especialistas" que possam dar voz a casos ou causas. O movimento em direção ao "empowerment" comunitário desloca o papel do profissional de representante para facilitador. ${ }^{(59)}$

Nesse ponto, cabe uma última consideração sobre a aquisição de competências profissionais. $\mathrm{O}$ debate sobre o tema ganha espaço a partir da década de 1980, quando então os modelos de produção e gerenciamento, relacionados às situações concretas de trabalho, trazem a necessidade de novos conhecimentos e novas habilidades. Vários autores descrevem dimensões políticas e conceituais envolvendo as competências, bem como seus reflexos na educação e currículos. ${ }^{(60),(61),(62)}$

Quanto à polissemia do termo, Saupe et al..$^{(63)}$ validaram a seguinte definição entre profissionais da saúde: entende-se por competência "o resultado de um conjunto de capacidades referidas aos conhecimentos, às habilidades e às atitudes que conferem ao profissional condições para desenvolver seu trabalho". No presente artigo, lança-se mão de definição semelhante, porém ampliada, proposta em documento recente sobre a educação dos profissionais de saúde no novo século: "Competência é o uso habitual e criterioso de comunicação, conhecimento, habilidades técnicas, raciocínio clínico, emoções, valores e reflexão na prática diária para o benefício do indivíduo e da comunidade a serem atendidos". (64) Importante

${ }^{58}$ BRASIL. MINISTÉRIO DA SAÚDE. Portaria n 687 MS/GM, de 30 de março de 2006. "Aprova a Política de Promoção da Saúde". Disponível em: <http://bvsms.saude.gov.br/bvs/publicacoes/ politica_nacional_promocao_saude_3ed.pdf>. Acesso em: 13 jul.2012.

${ }^{59}$ CARLISLE, S. op.cit.

${ }^{60}$ DELUIZ, N. Boletim Técnico SENAC, v. 27, n. 3, 2001.

${ }^{61}$ LIMA, V.V. Competência: distintas abordagens e implicações na formação de profissionais de saúde. Interface - Comunicação, Saúde, Educação, Botucatu, v. 9, n. 17, p. 369-79, 2005.

62 FRENK, J.; CHEN, L.; BHUTTA, Z.A.; COHEN, J.; CRISP, N.; EVANS, T.; FINEBERG, H. et al. Health professionals for a new century: transforming education to strengthen health systems in an interdependent world. The Lancet, London, v. 376, n. 9756, p. 1923-1958, 2010.

${ }^{63}$ SAUPE, R.; BENITO, G.A.V.; WENDHAUSEN, A.L.P.; CUTOLO, L.R.A. Conceito de Competência: validação por profissionais de saúde. Saúde em Revista, Piracicaba, v. 8, n. 18, p. 31-37, 2006.

${ }^{64}$ FRENK, J.; CHEN, L.; BHUTTA, Z.A.; COHEN, J.; CRISP, N.; EVANS, T.; FINEBERG, H. et al. op. cit. 
ressaltar, ainda, que a noção de competência extrapola a dimensão individual e engloba aspectos socioculturais e situacionais. Defende-se a construção de competências coletivas em prol da saúde da população, reconhecendo vantagens para o sistema de saúde, para as organizações envolvidas e para os profissionais.

Nessa linha, a advocacia em promoção da saúde aparece como competência central em iniciativas estrangeiras, como o documento da União Internacional de Promoção da Saúde e Educação para a Saúde (UIPES) e o projeto CompHP (Developing competencies and professional standards for health promotion capacity building in Europe). ${ }^{(65)}$ Espera-se que o promotor da saúde possua a competência de "Advogar, com e em nome de indivíduos, comunidades e organizações para melhorar a saúde e bem-estar e desenvolver a capacidade de ações de promoção da saúde".

Além das estratégias descritas, ponto comum que perpassa a discussão das competências (técnicas) para a advocacia da promoção da saúde relaciona-se com a habilidade de se comunicar efetivamente, por escrito, com o público alvo escolhido. Escrever é uma maneira ativa de encorajar profissionais a reconhecer os pedaços da informação e demandas que precisam ser articulados. Há relatos de experiências de cursos e programas de treinamento para o aprimoramento de tal habilidade, nos quais são desenvolvidas atividades práticas formais envolvendo um resumo de situação hipotética (o tema e o público alvo) e simulação de escrita de cartas para jornais, editores, políticos.

\section{Considerações finais}

Claro está que os debates não estão encerrados e, portanto, é conveniente entender o corpo de conhecimentos relacionado à advocacia em promoção da saúde. Com a trajetória aqui desenvolvida, pensa-se ter fundamentado como a identificação de conceitos, fundamentos e estratégias na advocacia em promoção da saúde é passo essencial para a sinergia com a prática profissional, de forma a contribuir para o enfrentamento das iniquidades em saúde no Brasil. Por outro lado, há necessidade de avanços na área de ensino e pesquisa para a construção e fortalecimento de tais práticas.

\section{Referências}

ANDRADE, R.D.; MELLO, D.F.; SILVA, M.A.I.; VENTURA, C.A.A. Advocacia em saúde na atenção à criança: revisão de literatura. Revista Brasileira de Enfermagem, Brasília, v. 64, n. 4, p. 738-744, 2011.

\footnotetext{
65 INTERNATIONAL UNION FOR HEALTH PROMOTION AND EDUCATION (IUHPE). The CompHP core competencies framework for health promotion handbook. 2011. 31 p. Disponível em: <http:// www.iuhpe.org/?page=614\&lang=en>. Acesso em: 13 jul. 2012.
} 
ASSOCIAÇÃO PARANAENSE DA PARADA DA DIVERSIDADE - APPAD. Guia de advocacy e prevenção em HIV/AIDS: gays e outros homens que fazem sexo com homens. Curitiba. 2009. 101p. Disponível em: <http://www.abglt.org.br/docs/ interagir_guia.pdf>. Acesso em: 12 mar. 2012.

AYRES, J.R.C.M. Norma e formação: horizontes filosóficos para as práticas de avaliação no contexto da promoção da saúde. Ciência \& Saúde Coletiva, Rio de Janeiro, v. 9, n. 3, p. 583-592, 2004.

BRASIL. Instituto Nacional do Câncer INCA. Observatório da política nacional de controle do tabaco. Disponível em: <http://www2.inca.gov.br/wps/wcm/connect/ observatorio_controle_tabaco/site/status_politica/a_politica_nacional>. Acesso em: 12 mar. 2012.

MINISTÉRIO DA SAÚDE. Política nacional de Promoção da Saúde. Disponível em: <http://bvsms.saude.gov.br/bvs/publicacoes/politica_nacional_ promocao_saude_3ed.pdf>. Acesso em: 13 jul. 2012.

Secretaria de Políticas de Saúde. Projeto Promoção da Saúde. As Cartas da Promoção da Saúde. Ministério da Saúde, Secretaria de Políticas de Saúde, Projeto Promoção da Saúde. - Brasília: Ministério da Saúde, 2002. Disponível em: <http://bvsms.saude.gov.br/bvs/publicacoes/cartas_promocao. pdf>. Acesso em: 13 jul. 2012.

CAIRA, N.M.; LACHENMAYR, S.; SHEINFELD, J.; GOODHART, F.W.; CANCIALOSI, L.; LEWIS, C. The health educator's role in advocacy and policy: principles, processes, programs, and partnerships. Health Promotion Practice, Thousand Oaks, v. 4, p. 303-313, 2003.

CANDEIAS, N.M.F. Conceitos de educação e de promoção em saúde: mudanças individuais e mudanças organizacionais. Revista de Saúde Pública, São Paulo, v. 31, n. 2, p 209-213, abr. 1997.

CANEL, R.C.; CASTRO, C.G.J. A advocacia em saúde como uma estratégia para a promoção da Saúde. Revista de Direito Sanitário, São Paulo, v. 9, n.1, p. 74-85, mar./jun. 2008.

CARLISLE, S. Health promotion, advocacy and health inequalities: a conceptual framework. Health Promotion International, Oxford, v. 15, n. 4, p. 369-376, 2000.

CARTA DE OTTAWA. Documento resultante da Primeira Conferência Internacional Sobre Promoção da Saúde de novembro de 1986. Disponível em: <http://www.opas.org.br/promocao/uploadArq/Ottawa.pdf>. Acesso em: 12 de mar. 2012.

CARVALHO, S.R. Os múltiplos sentidos da categoria "empowerment" no projeto de promoção à saúde. Cadernos de Saúde Pública, Rio de Janeiro, v. 20, n. 4, p. 1088-1095, jul./ago. 2004. 
CHIEFI, A.L.; BARATA, R.B. Judicialização da política pública de assistência farmacêutica e eqüidade. Cadernos de Saúde Pública, Rio de Janeiro, v. 25, n. 8, p. 1839-1849, jun. 2009.

COALITION QUÉBÉCOI POUR LE CONTRÔLE DU TABAC. Fazendo pesquisa para advocacy: a arte de converter o impossível no inevitável - e com baixo custo. 2006. 44p. Disponível em: <http://actbr.org.br/uploads/conteudo/49_860_TABA. pdf>. Acesso em: 12 mar. 2012.

DALLARI, S.G.; BARBER-MADDEN, R.; TORRES-FERNANDES, M.C.; SHUQUAIR, N.S.M.S.A.Q; WATANABE, H.A. Advocacia em saúde no Brasil contemporâneo. Revista de Saúde Pública, São Paulo, v. 30 n. 6, p. 592-601, abr. 1996.

DELUIZ, N. Boletim Técnico SENAC, v. 27, n. 3, 2001.

DEVLIN-FOLTZ, FAGEN M.C.; REED, E.; MEDINA, R.; NEIGER, B.L. Advocacy evaluation: challenges and emerging trends. Health Promotion Practice, Thousand Oaks, v. 13, n. 5, p. 581-586, 2012.

FEUERWERKER, L.C.M. O papel das equipes de saúde na advocacia em saúde. Saúde em Debate, Rio de Janeiro, v. 45, p. 47-25, 1994.

FRENK, J.; CHEN, L.; BHUTTA, Z.A.; COHEN, J.; CRISP, N.; EVANS, T.; FINEBERG, $\mathrm{H}$. et al. Health professionals for a new century: transforming education to strengthen health systems in an interdependent world. The Lancet, London, v. 376, n. 9756, p. 1923-1958, 2010.

GALER-UNTI, R.A. Advocacy 2.0: advocating in the digital age. Health Promotion Practice, Thousand Oaks, v. 11, n. 6, p. 784-787, 2010.

; TAPPE, M.K.; LACHENMAYR, S. Advocacy 101: Getting Started in Health Education Advocacy. Health Promotion Practice, Thousand Oaks, v. 5, p. 280-288, 2004.

GIRARDI, S. N.; FERNANDES JUNIOR, H.; CARVALHO, C. L. A regulamentação das profissões de saúde no Brasil. Espaço para Saúde, Londrina, v. 2, n. 1. Disponível em: <http://www.ccs.uel.br/espaçoparasaude/v2n1/RPSB.htm>. Acesso em: 13 jul. 2012.

HOGUE, M-C.B.; DORAN, E.; HENRY, D.A. A prompt to the web: the media and health information seeking behaviour. PLOS ONE, v. 7, n. 4, p. e34314, 2012.

INTERNATIONALUNIONFORHEALTHPROMOTIONANDEDUCATION (IUHPE). The CompHP core competencies framework for health promotion handbook. 2011. 31p. Disponível em: <http://www.iuhpe.org/?page=614\&lang=en>. Acesso em: 13 jul. 2012. 
JOHANSSON, H.; WEINEHALL, L.; EMMELIN, M. It depends on what you mean: a qualitative study of Swedish health professionals'view on health and health promotion. BMC Health Services Research, v. 9, 2009.

LIMA, V.V. Competência: distintas abordagens e implicações na formação de profissionais de saúde. Interface: comunicação, saúde, educação, Botucatu, v. 9, n.17, p. 369-379, 2005.

MACHADO, F.R.S. Contribuições ao debate da judicialização da saúde no Brasil. Revista de Direito Sanitário, São Paulo v. 9, n. 2, p. 73-91, jul./out. 2008.

ORGANIZAÇÃO DAS NAÇÕES UNIDAS. Pacto Internacional dos Direitos Econômicos, Sociais e Culturais. Disponível em: <http://www2.mre.gov.br/ dai/m_591_1992.htm>. Acesso em: 12 mar. 2012.

PUBLIC HEALTH ALLIANCE FOR THE ISLAND OF IRELAND (PHAII). Public Health Advocacy Toolki, Belfast, 2007. 68 p. Disponível em: <http://www.phaii. org/>. Acesso em: 11 jan. 2012.

RODNEY, M.; CLASEN, C.; GOLDMAN, G.; MARKERT, R.; DEANE, D. Three evaluation methods of a community health advocate program. Journal of Community Health, v. 23, n. 5, Oct. 1998.

ROTHER, E.T. Revisão sistemática x revisão narrativa. Acta Paul Enferm., São Paulo, v. 20, n. 2, p. v-vi 2007.

SAUPE, R.; BENITO, G.A.V.; WENDHAUSEN, A.L.P.; CUTOLO, L.R.A. Conceito de competência: validação por profissionais de saúde. Saúde em Revista, Piracicaba, v. 8, n. 18, p. 31-37, 2006.

SHILTON, T. Advocacy for physical activity-from evidence to influence. Global Health Promotion, v. 13, n. 2, p. 118-126, 2006.

SOARES, M.C. Internet e saúde: possibilidades e limitações. Textos de la CiberSociedad, 4. Temática variada. 2004. Disponível em: <http://www. cibersociedad.net/textos/articulo.php?art=51>. Acesso em: 15 out. 2012.

TORRONTEGUY, M.A.A.; RAUPP, L. Obstáculos políticos à advocacia sanitária no brasil: a pesquisa com células-tronco embrionárias. Revista de Direito Sanitário, São Paulo, v. 9, n. 1, p. 86-106, mar.jun. 2008.

WORLD HEALTH ORGANIZATION (WHO). An International Framework Convention for Tobacco Control. Fact Sheet $n^{\circ} 160$. Disponível em: <http://www. who.int/inf-fs/en/fact160.html>. Tradução disponível em: <http://actbr.org.br/pdfs/ CQCT.pdf>. Acesso em: 13 jul. 2012.

Rio Political Declaration on Social Determinants of Health. Rio de Janeiro, Brazil, 21 Oct. 2011. 7p. Disponível em: <http://cmdss2011.org/site/ wp-content/uploads/2011/10/Final-draft-EN-Declaration_20-October-FI.pdf>. Acesso em: 05 out. 2012. 
ZIONI, F. A advocacia em saúde e sua contribuição à democracia. Revista de Direito Sanitário, São Paulo, v. 9, n. 1, p. 70-73, mar./jun. 2008.

ANEXO 1 - Websites para informações adicionais sobre assuntos relacionados à advocacia em promoção da saúde.

\begin{tabular}{|c|c|c|}
\hline Endereço do site & Organização & Descrição \\
\hline $\begin{array}{l}\text { http://www.cepedisa. } \\
\text { org.br/apresentacao. } \\
\text { shtml }\end{array}$ & $\begin{array}{l}\text { Centro de Estudos e } \\
\text { Pesquisas de Direito } \\
\text { Sanitário - Cepedisa }\end{array}$ & $\begin{array}{l}\text { Divulga informações, nacionais } \\
\text { e internacionais, relacionadas ao } \\
\text { direito sanitário, incluindo trabalhos } \\
\text { acadêmicos, legislação, doutrina, } \\
\text { jurisprudência, entre outros. }\end{array}$ \\
\hline $\begin{array}{l}\text { http://conselho. } \\
\text { saude.gov.br/ }\end{array}$ & & $\begin{array}{l}\text { Instância máxima de deliberação } \\
\text { do Sistema Único de Saúde, de } \\
\text { caráter permanente e deliberativo, } \\
\text { composto por representantes } \\
\text { de entidades e movimentos } \\
\text { representativos de usuários, } \\
\text { entidades representativas de } \\
\text { trabalhadores da área da saúde, } \\
\text { governo e prestadores de serviços } \\
\text { de saúde. }\end{array}$ \\
\hline $\begin{array}{l}\text { http://observatorio. } \\
\text { fiocruz.br/start.php }\end{array}$ & $\begin{array}{l}\text { Fundação Oswaldo Cruz } \\
\text { Escola Nacional de Saúde } \\
\text { Pública Sergio Arouca } \\
\text { Consultoria Legislativa do } \\
\text { Senado Federal } \\
\text { Consultoria Legislativa da } \\
\text { Câmara dos Deputados } \\
\text { Cepedisa e Ministério da } \\
\text { Saúde }\end{array}$ & $\begin{array}{l}\text { Mapeia, analisa, discute e difunde } \\
\text { as propostas sobre saúde que } \\
\text { tramitam no Congresso Nacional. }\end{array}$ \\
\hline $\begin{array}{l}\text { http://portalsaude. } \\
\text { saude.gov.br/ } \\
\text { portalsaude/area/320/ } \\
\text { legislacao.html }\end{array}$ & Ministério da Saúde & Reúne legislação vigente do SUS. \\
\hline $\begin{array}{l}\text { http://portal.saude. } \\
\text { gov.br/portal/saude/ } \\
\text { profissional/area. } \\
\text { cfm?id_area=1484 }\end{array}$ & Ministério da Saúde & $\begin{array}{l}\text { Reúne material sobre promoção } \\
\text { da saúde. }\end{array}$ \\
\hline $\begin{array}{l}\text { http://www.ans. } \\
\text { gov.br/index.php/ } \\
\text { legislacao/busca-de- } \\
\text { legislacao }\end{array}$ & $\begin{array}{l}\text { Agência Nacional de } \\
\text { Saúde Suplementar }\end{array}$ & $\begin{array}{l}\text { Traz legislação regulamentada } \\
\text { pela agência reguladora } \\
\text { vinculada ao Ministério da Saúde } \\
\text { responsável pelo setor de planos } \\
\text { de saúde no Brasil. }\end{array}$ \\
\hline
\end{tabular}

Continua

R. Dir. sanit., São Paulo, v. 14, n. 1, p. 34-59, mar./jun. 2013 
Continuação Anexo 1

\begin{tabular}{|c|c|c|}
\hline Endereço do site & Organização & Descrição \\
\hline $\begin{array}{l}\text { http://www.who.int// } \\
\text { school_youth_health/ } \\
\text { resources/en/ }\end{array}$ & $\begin{array}{l}\text { Organização Mundial da } \\
\text { Saúde }\end{array}$ & $\begin{array}{l}\text { Reúne recursos e ferramentas que } \\
\text { apoiam programas de prevenção } \\
\text { e promoção em escolas e/ou } \\
\text { voltados para adolescentes (em } \\
\text { inglês). }\end{array}$ \\
\hline $\begin{array}{l}\text { http://www2.inca.gov. } \\
\text { br/wps/wcm/connect/ } \\
\text { observatorio_ } \\
\text { controle_tabaco/site/ } \\
\text { home }\end{array}$ & $\begin{array}{l}\text { Instituto Nacional do } \\
\text { Câncer }\end{array}$ & $\begin{array}{l}\text { Acompanhamento da adoção } \\
\text { das políticas governamentais } \\
\text { voltadas para o cumprimento } \\
\text { da Convenção-Quadro para o } \\
\text { Controle do Tabaco. Acesso a } \\
\text { pesquisas, publicações, legislação } \\
\text { e outras informações sobre o } \\
\text { controle do tabagismo. }\end{array}$ \\
\hline $\begin{array}{l}\text { http://actbr.org. } \\
\text { br/uploads/ } \\
\text { conteudo/49_860_ } \\
\text { TABA.pdf }\end{array}$ & $\begin{array}{l}\text { Aliança Contra o Tabaco } \\
\text { (ACT) }\end{array}$ & $\begin{array}{l}\text { Manual traduzido pela ACT sobre } \\
\text { a pesquisa para a advocacia em } \\
\text { saúde, relacionada ao tabaco. }\end{array}$ \\
\hline
\end{tabular}

